\title{
Estimativas de parâmetros genéticos para características reprodutivas de suínos
}

[Genetic parameters estimates for reproductive traits in swine]

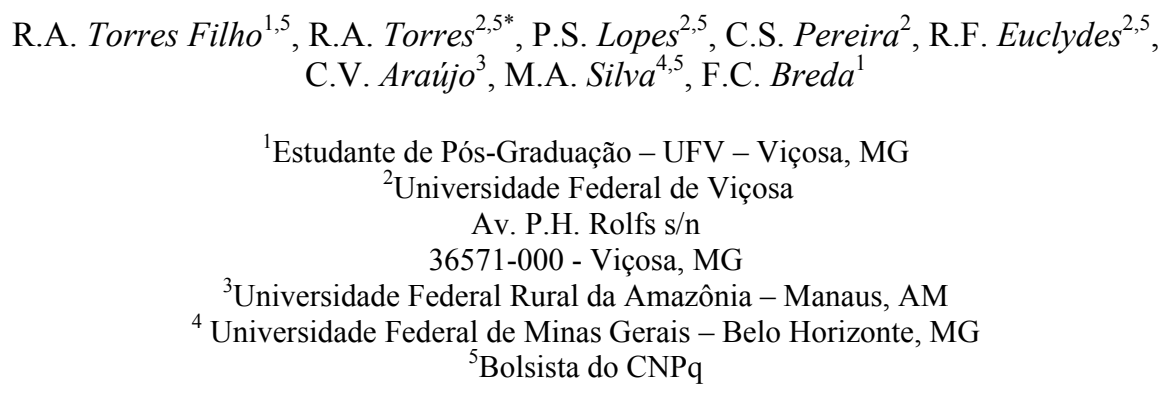

\section{RESUMO}

Foram utilizados dados referentes à idade da fêmea no primeiro parto (IPP), número total de leitões nascidos (NLN), número de leitões nascidos vivos (NLNV) e peso da leitegada no nascimento (PLV) para estimar parâmetros genéticos e fenotípicos de características reprodutivas de suínos da raça Large White. Variâncias e covariâncias genéticas e fenotípicas foram estimadas pelo método da máxima verossimilhança restrita (REML), usando na análise da IPP o modelo que incluiu os efeitos genéticos direto e materno e comum de leitegada. Para NLN, NLNV e PLV, o modelo incluiu apenas efeito genético direto. As herdabilidades para os efeitos genéticos aditivos direto variaram de 0,17 a 0,34 . A maioria das características apresentou baixa herdabilidade, sugerindo a necessidade de estratégias de seleção que incluam informações de família para obtenção de progresso genético. As correlações genéticas indicaram que IPP está associada às demais características estudadas e que a resposta em NLN pode ser obtida por meio da seleção em NLNV.

Palavras-chave: suíno, primeiro parto, número de leitões, peso da leitegada, correlação, herdabilidade

\begin{abstract}
Data on age at first farrowing (IPP), total number of piglets born (NLN), number of piglets born alive $(N L N V)$, and birth litter weight (PLV) were used to estimate genetic and phenotypic parameters of reproductive traits in Large White breed. Genetic and phenotypic variances and covariances were estimated by Restricted Maximum Likelihood method (REML). The model to analyze IPP included direct genetic effect, maternal effect and common litter effect, and the model to analyze NLN, NLNV and PLV included only direct genetic effect. Heritability estimates for direct additive genetic effects ranged from 0.17 to 0.34. Favorable genetic correlations between IPP and the other traits studied were observed. The low heritability estimates for most traits suggest that efficient selection strategies must be sought to increase genetic gain and that response for NLN may be obtained by indirect selection on NLNV.
\end{abstract}

Keywords: swine, first farrowing, litter size, litter weight, genetic correlation, heritability

Apoio financeiro: $\mathrm{CNPq}$

Recebido para publicação em 19 de março de 2004

Recebido para publicação, após modificações, em 10 de setembro de 2004

*Autor para correspondência (corresponding author)

E-mail: rtorres@ufv.br 


\section{INTRODUÇÃO}

Em suínos, as características reprodutivas são consideradas como sendo da porca, e sua importância está relacionada ao aumento da eficiência do sistema, pois quanto melhor o desempenho reprodutivo do rebanho, menor será o custo de manutenção por matriz. As estimativas de herdabilidade das características reprodutivas nos animais são normalmente baixas, e a variabilidade existente em tais estimativas é atribuída, principalmente, a fatores não genéticos e genéticos não aditivos.

Segundo Rydhmer (2000), no aspecto econômico, o tamanho da leitegada é a característica reprodutiva mais importante e já é considerada em vários programas de melhoramento. O peso ao nascer é um dos fatores que mais influem na sobrevivência do leitão e está diretamente relacionado ao tamanho da leitegada, razão porque um equilíbrio entre essas características é sempre desejável. Alguns autores citaram o peso do leitão no nascimento como o fator isolado mais importante para a sobrevivência (Roehe e Kalm, 1999), para o peso da leitegada na desmama (Winters et al., 1947) e para o desempenho na vida do animal (Rydhmer et al., 1989).

De acordo com Freitas (1998), a redução da idade à puberdade significa menor idade no acasalamento e no primeiro parto, reduzindo a idade de entrada das fêmeas no plantel, o que representa menor custo de produção de leitões. A herdabilidade dessa característica é de $30 \%$, e as correlações genéticas e fenotípicas são favoráveis à taxa de crescimento. Assim, há variação aditiva e efeitos pleiotrópicos abundantes para mudanças na idade à puberdade.

Irgang et al. (1997), ao trabalharem com dados das Associações de Criadores de Suínos dos estados do Rio Grande do Sul, Santa Catarina e Paraná, concluíram que os ganhos genéticos no número de leitões nascidos vivos por leitegada, em fêmeas Landrace e Large White, foram positivos, porém pequenos, o que indica pouca intensidade de seleção aplicada ao melhoramento dessa característica. Sugeriram, então, a adoção de técnicas modernas de avaliação genética e esforço contínuo de seleção pelos criadores e melhoristas para obtenção de maiores ganhos genéticos.
A inclusão de características reprodutivas no melhoramento genético de suínos ainda é uma dúvida por parte de muitos melhoristas, em razão das baixas herdabilidades e de sua expressão ser limitada a animais adultos (Lopes et al., 1998).

O presente trabalho teve como objetivos estimar os componentes de variância e covariância para os efeitos genético direto e materno e as correlações genéticas de características reprodutivas de suínos.

\section{MATERIAL E MÉTODOS}

Os dados utilizados, a metodologia adotada para obter as estimativas dos componentes de variância e os parâmetros genéticos foram descritos por Torres Filho et al. (2004).

As características avaliadas foram idade da fêmea ao primeiro parto (IPP), número total de leitões nascidos (NLN), número de leitões nascidos vivos (NLNV) e peso da leitegada ao nascimento (PLV). Somente foram pesados os animais nascidos vivos.

O modelo adotado para análise da idade da porca ao primeiro parto nesta população incluiu o efeito genético direto do animal, o efeito materno e o efeito comum de leitegada. Para as características de leitegada, o modelo incluiu apenas o efeito genético direto do animal.

Como efeitos fixos, foram utilizados os grupos contemporâneos, formados pela combinação da semana do ano $\left(1=48^{\mathrm{a}}\right.$ à $8^{\mathrm{a}}$ semana; $2=9^{\mathrm{a}}$ à $21^{\mathrm{a}}$ semana; $3=22^{\text {a }}$ à $34^{\text {a }}$ semana; e $4=35^{\text {a }}$ à $47^{\text {a }}$ semana) com o ano do parto. Os efeitos linear e quadrático da covariável idade da porca no parto foram incluídos nos modelos para número total de leitões nascidos, número de leitões nascidos vivos e peso da leitegada ao nascer.

\section{RESULTADOS E DISCUSSÃO}

As estimativas de variâncias genéticas aditivas diretas, de variância residual, de variância fenotípica e de covariâncias diretas, residuais e fenotípicas, em análise de característica única e bicaracterística, são apresentadas na Tab. 1 . 
Tabela 1. Estimativas de variâncias genéticas aditivas diretas $\left(\hat{\sigma}_{\mathrm{d}}^{2}\right)$, de variância residual $\left(\hat{\sigma}_{\mathrm{e}}^{2}\right)$, de variância fenotípica $\left(\hat{\sigma}_{\mathrm{f}}^{2}\right)$, e de covariâncias diretas $\left(\hat{\sigma}_{\mathrm{d}_{1,2}}\right)$, residuais $\left(\hat{\sigma}_{\mathrm{e}_{1,2}}\right)$ e fenotípicas $\left(\hat{\sigma}_{\mathrm{f}_{1,2}}\right)$, obtidas em análise de característica única e em análise bicaracterística ${ }^{1,2}$

\begin{tabular}{|c|c|c|c|c|c|c|}
\hline Característica & $\hat{\sigma}_{\mathrm{d}}^{2}$ & $\hat{\sigma}_{\mathrm{d}_{1,2}}$ & $\hat{\sigma}_{\mathrm{e}}^{2}$ & $\hat{\sigma}_{e_{1,2}}$ & $\hat{\sigma}_{f}^{2}$ & $\hat{\sigma}_{f_{1,2}}$ \\
\hline \multicolumn{7}{|c|}{ Idade da porca ao primeiro parto (IPP) } \\
\hline Unicaracterística $^{3}$ & 107,49838 & - & 185,58054 & - & 315,85678 & - \\
\hline NLN & 110,12366 & $-3,02342$ & 182,76069 & 4,90563 & 316,68209 & 1,88221 \\
\hline NLNV & 110,34332 & $-2,77052$ & 183,02575 & 4,34924 & 316,74381 & 1,57872 \\
\hline PLV & 107,28717 & $-1,32123$ & 185,51381 & 5,24337 & 315,95644 & 3,92214 \\
\hline \multicolumn{7}{|c|}{ Número total de leitões nascidos (NLN) } \\
\hline Unicaracterística & 2,30751 & - & 8,06328 & - & 10,37078 & - \\
\hline IPP & 2,31005 & $-3,02342$ & 8,06251 & 4,90563 & 10,37256 & 1,88221 \\
\hline NLNV & 2,33108 & 2,15921 & 8,04117 & 7,62849 & 10,37224 & 9,78770 \\
\hline PLV & 2,29484 & 2,00699 & 8,07026 & 8,20650 & 10,36509 & 10,21349 \\
\hline \multicolumn{7}{|c|}{ Número de leitões nascidos vivos (NLNV) } \\
\hline Unicaracterística & 1,93971 & - & 8,22384 & - & 10,16355 & - \\
\hline IPP & 1,94381 & $-2,77052$ & 8,22318 & 4,34924 & 10,16699 & 1,57872 \\
\hline NLN & 2,01588 & 2,15921 & 8,17730 & 7,62849 & 10,19319 & 9,78770 \\
\hline PLV & 1,91845 & 1,76301 & 8,23711 & 9,15789 & 10,15556 & 10,92090 \\
\hline \multicolumn{7}{|c|}{ Peso da leitegada ao nascimento (PLV) } \\
\hline Unicaracterística & 2,81916 & - & 13,29423 & - & 16,11339 & - \\
\hline IPP & 2,80324 & $-1,32123$ & 13,30482 & 5,24337 & 16,10806 & 3,92214 \\
\hline NLN & 2,86059 & 2,00699 & 13,27179 & 8,20650 & 16,13238 & 10,21349 \\
\hline NLNV & 2,79339 & 1,76301 & 13,31028 & 9,15789 & 16,10367 & 10,92090 \\
\hline
\end{tabular}

As estimativas de variâncias atribuídas aos efeitos genéticos aditivos diretos e residuais, obtidas em análise bicaracterística, foram semelhantes às obtidas em análise unicaracterística. Considerando que essas estimativas de componentes de variância são influenciadas pela unidade da medida adotada, as discussões dos parâmetros genéticos foram realizadas com base apenas nas estimativas de herdabilidade, correlações genéticas e nos coeficientes $\mathrm{c}^{2}$.

Estimativas de herdabilidades direta, materna e total, de correlações entre os efeitos aditivos direto e materno, da proporção atribuída aos efeitos comuns de leitegada e dos respectivos erros-padrão das características estudadas são apresentadas na Tab. 2.

Tabela 2. Herdabilidades para efeitos genéticos aditivos direto $\left(\hat{\mathrm{h}}_{\mathrm{d}}^{2}\right)$ e materno $\left(\hat{\mathrm{h}}_{\mathrm{m}}^{2}\right)$, correlações entre efeitos aditivos direto e materno $\left(\mathrm{r}_{\mathrm{dm}}\right)$, herdabilidade total $\left(\hat{\mathrm{h}}_{\mathrm{T}}^{2}\right)$, proporção atribuída aos efeitos comuns de leitegada $\left(\hat{c}^{2}\right)$ e estimativas dos respectivos erros-padrão (EP)

\begin{tabular}{lccccc}
\hline \multicolumn{1}{c}{ Característica } & $\hat{\mathrm{h}}_{\mathrm{d}}^{2} \pm \mathrm{EP}$ & $\hat{\mathrm{h}}_{\mathrm{m}}^{2} \pm \mathrm{EP}$ & $\mathrm{r}_{\mathrm{dm}} \pm \mathrm{EP}$ & $\hat{\mathrm{h}}_{\mathrm{T}}^{2}$ & $\hat{\mathrm{c}}^{2} \pm \mathrm{EP}$ \\
\hline IPP & $0,34 \pm 0,086$ & $0,07 \pm 0,046$ & $-0,72 \pm 0,589$ & 0,21 & $0,11 \pm 0,042$ \\
NLN & $0,22 \pm 0,022$ & -- & -- & 0,22 & -- \\
NLNV & $0,19 \pm 0,022$ & - & -- & 0,19 & - \\
PLV & $0,17 \pm 0,21$ & -- & -- & 0,17 & -- \\
\hline
\end{tabular}

IPP = Idade da porca ao primeiro parto; NLN = número total de leitões nascidos; NLNV = número de leitões nascidos vivos; PLV = peso da leitegada ao nascimento. 
$\mathrm{O}$ valor de $\hat{\mathrm{h}}_{\mathrm{d}}^{2}$ da idade da porca ao primeiro parto indica que essa característica apresentaria ganho genético como resposta à seleção massal. Embora Irgang (1985) tenha encontrado, para idade da fêmea na primeira concepção e na primeira cobertura, estimativas de herdabilidade de 0,47 e 0,48 , respectivamente, a herdabilidade materna $\left(\hat{h}_{m}^{2}=0,07\right)$ obtida neste trabalho é semelhante às encontradas para diferentes características reprodutivas.

A estimativa do efeito comum de leitegada para IPP destaca a importância de sua inclusão no modelo de avaliação da IPP, uma vez que foi responsável por $11 \%$ da variação fenotípica total.

Irgang et al. (1994) destacaram que as herdabilidades do tamanho da leitegada em torno de 0,10 foram obtidas tanto por regressão mãefilha quanto pela correlação intraclasse de meioirmãos paternos. Dentre as estimativas obtidas por meio de modelo animal, relatadas em estudos mais recentes, destacam-se as de Chen et al. (2003), que variaram de 0,08 a 0,10 , as de Pires et al. (2000), de 0,16 a 0,22, e a de Kaufmann et al. (2000), de 0,22. Assim, as estimativas de herdabilidade para número total de leitões nascidos e nascidos vivos obtidas neste estudo $(0,22$ e 0,19 , respectivamente) são elevadas quando comparadas ao valor de 0,10 , porém estão dentro das amplitudes obtidas por Kaufman et al. (2000) e Pires (1999). Kaufmann et al. (2000) sugeriram como uma das possíveis razões da maior magnitude da estimativa a homogeneidade das condições ambientais (manejo e alimentação) a que os animais foram submetidos.

Embora Perez-Encizo e Gianola (1992) tenham ressaltado que as baixas herdabilidades e o risco de aumento da endogamia sejam empecilhos na seleção para prolificidade de suínos, Holl e Robinson (2003), ao avaliarem os resultados de nove gerações de seleção em uma linha de suínos composta (Landrace x Large White), concluíram que o tamanho da leitegada pode ser aumentado por meio da seleção direta. Desse modo, confirma-se a possibilidade de se obterem, mediante seleção, resultados satisfatórios do tamanho de leitegada na linha de suínos da raça Large White.

A estimativa de herdabilidade direta do peso da leitegada ao nascimento foi elevada em relação ao valor de 0,11 , obtido por Upnomoor (1984), e dentro das amplitudes de 0,00 a 0,24 e 0,17 a 0,21, obtidas por Alves (1986) e Pires (1999), respectivamente.

As estimativas de correlações genéticas aditivas diretas encontram-se na Tab. 3.

Tabela 3. Estimativas de correlações genéticas aditivas diretas, obtidas de características reprodutivas, em análise bicaracterística

\begin{tabular}{|c|c|c|c|c|}
\hline \multirow{2}{*}{ Característica } & \multicolumn{4}{|c|}{ Correlação genética } \\
\hline & IPP & NLN & NLNV & PLV \\
\hline Idade da porca no primeiro parto(dias) (IPP) & 1 & $-0,19$ & $-0,19$ & $-0,08$ \\
\hline Número total de leitões nascidos (NLN) & & 1 & 1 & 0,78 \\
\hline Número de leitões nascidos vivos (NLNV) & & & 1 & 0.76 \\
\hline Peso da leitegada no nascimento (kg) (PLV) & & & & 1 \\
\hline
\end{tabular}

As correlações genéticas entre idade da porca ao primeiro parto e as características número de leitões nascidos e peso da leitegada ao nascimento foram negativas, sendo que entre IPP e PLV foi a de menor magnitude. Elas indicam que fêmeas que tiveram o primeiro parto mais jovens tenderam a ser mais prolíferas. Estes resultados podem ser atribuídos aos critérios de seleção adotados no programa de melhoramento.
O alto valor da correlação genética entre número de leitões total e nascidos vivos foi semelhante à estimativa de 0,93, obtida por Holl e Robison (2003). Assim, na avaliação genética dos animais, pode-se utilizar a informação de apenas uma das características no critério de seleção.

As correlações entre peso da leitegada no nascimento e número de leitões total e nascidos vivos foram positivas e de alta magnitude, razão 
pela qual partos mais numerosos tendem a apresentar leitegadas mais pesadas. Os valores encontrados neste trabalho estão dentro da amplitude de 0,61 a 0,89 encontrada por Pires et al. (2000).

Kaufmann et al. (2000), ao estudarem a relação entre tamanho da leitegada e peso do leitão ao nascimento e ao desmame, encontraram associação negativa entre as características e ressaltaram que a seleção para tamanho da leitegada poderia levar à tendência negativa do efeito genético materno sobre o peso individual ao nascimento e ao desmame. Considerando que tamanho da leitegada tem valor econômico elevado na suinocultura e que o baixo peso ao nascimento aumenta a mortalidade pré-desmame e diminui o crescimento pós-parto, Kaufmann et al. (2000) afirmaram que a seleção combinada para tamanho da leitegada e peso individual poderia ser necessária para aumentar a produtividade da porca.

Correlações desfavoráveis entre duas características podem atrasar o progresso genético, caso sejam selecionadas separadamente em programas de melhoramento genético, razão pela qual devem ser avaliadas por meio de metodologias ou procedimentos multidimensionais. O número de características incluídas na análise deve ser considerado, primeiro para evitar problemas computacionais e segundo porque o aumento do número de características no critério de seleção acarreta menor ganho genético por característica. Considerando que a correlação genética entre número de leitões nascidos vivos e peso da leitegada foi de 0,76 , e que, em razão da crescente competitividade do setor, as linhas fêmeas não são selecionadas apenas para características reprodutivas, deve-se adotar como alternativa a inclusão do tamanho de leitegada no critério de seleção e o monitoramento do peso individual ao nascimento e à desmama.

\section{CONCLUSÕES}

A idade da porca no primeiro parto apresentou a maior magnitude de herdabilidade entre as características avaliadas e correlação favorável com as demais características. A correlação genética entre número total de leitões nascidos e nascidos vivos indica que apenas uma das duas é suficiente para ser selecionada em programas de melhoramento.

\section{AGRADECIMENTO}

À empresa SADIA S.A., pela concessão dos dados que possibilitaram a execução deste trabalho.

\section{REFERÊNCIAS BIBLIOGRÁFICAS}

ALVES, R.G.O. Estudo genético de características em suínos e avaliação de curvas de crescimento em cruzamentos dialélicos. 1986. 129f. Tese (Doutorado) - Departamento de Zootecnia, Universidade Federal de Viçosa, Viçosa, MG.

CHEN, P.; BAAS, T.J.; MABRY, J.W. et al. Genetic parameters and trends for litter traits in U.S. Yorkshire, Duroc, Hampsire, and Landrace pigs. J. Anim. Sci., v.81, p.46-53, 2003.

FREITAS, R.T.F. Situação atual e perspectiva do melhoramento de suínos. In: CONGRESSO NACIONAL DOS ESTUDANTES DE ZOOTECNIA, 1998, Viçosa. Anais... Viçosa: UFV, 1998. p.461-471.

HOLL, J.W.; ROBISON, O.W. Results from nine generations of selection for increased litter size in swine. J. Anim. Sci., v.81, p.624-629, 2003.

IRGANG, R.. Estimativas de herdabilidade para características que compões a produtividade anual de leitões por porca. Concórdia: EMBRAPA-CNPSA, 1985. 4p. (Comunicado Técnico, 81).

IRGANG, R.; FÁVERO, J.A.; KENNEDY, B.Y. Genetic parameters for litter size of diferent parities in Duroc, Landrace and Large White sows. J. Anim. Sci., v.72, p.2237-2246, 1994.

IRGANG, R.; FÁVERO, J.A.; MEDEIROS, G. Tendências fenotípicas e genéticas do número de leitões nascidos vivos em porcas Landrace e Large White. In: REUNIÃO ANUAL DA SOCIEDADE BRASILEIRA DE ZOOTECNIA, 34., 1997, Juiz de Fora. Anais... Juiz de Fora, SBZ, 1997. p.320-323 (Resumo).

KAUFMANN, D.; HOFER, A.; BIDANEL, J.P. et al. Genetic parameters for individual birth and 
weaning weight for litter size of Large White pigs. J. Anim. Breed. Genet., v.117, p.121-128, 2000.

LOPES. P.S.; FREITAS, R.T.F.; FERREIRA, A.S. Melhoramento de suínos. Viçosa:UFV, 1998. 39p. (Caderno Didático, 37).

PEREZ-ENCIZO, M.; GIANOLA, D. Estimates of genetic parameters for litter size in six strains of Iberian pigs. Livest. Prod. Sci., v.32, p.283293, 1992.

PIRES, A.V. Avaliação genética de características reprodutivas em suínos. 1999. 83f. Dissertação (Mestrado) - Departamento de Zootecnia, Universidade Federal de Viçosa, Viçosa, MG.

PIRES, A.V.; LOPES, P.S.; TORRES, R.A. et al. Estimação de parâmetros genéticos de características reprodutivas em suínos. Rev. Bras. Zootec., v.29, p.1698-1705, 2000.

ROEHE, R.; KALM, E. Risk factors and genetic variance components of pre-weaning mortality in piglets. In: ANNUAL MEETING OF THE European Association of Animal Production, 50., 1999, Zürich. Proceedings... Zürich: EAAP, 1999 .
RYDHMER, L. Genetics of sow reproduction, including puberty, oestrus, pregnancy, farrowing and lactation. Livest. Prod. Sci., v.66, p.1-12, 2000.

RYDHMER, L.; ELIASSON, L.; STERN, S. et al. Effects of piglet weight and fraternity size on performance, puberty and farrowing results. Acta Agric. Scand., v.39, p.397-406, 1989.

TORRES FILHO, R.A.; TORRES, R.A.; LOPES, P.S. et al. Avaliação de modelos para estimação de componentes de (co)variância em características de desempenho e reprodutivas em suínos. Rev. Bras. Zootec., v.33, p.350-357, 2004.

UPNMOOR, I. Influência de fatores genéticos e de ambiente sobre a produtividade de suínos mestiços Landrace e Large White. 1984. 180f. Dissertação (Mestrado) - Departamento de Zootecnia, Universidade Federal de Viçosa, Viçosa, MG.

WINTERS, L.M.; CUMMINGS, J.N.; STEWART, H.A. A study of factors affecting survival from bith to weaning and total weaning weigth of the litter in swuine. J. Anim. Sci., v.6, p.288-296, 1947. 